\title{
Mobile Indoor Localization in Museum Environment: An Experimentation in FIBAC Project
}

\author{
Simona Acanfora \\ Poste Italiane \\ Information Technology \\ P.zza Matteotti 3, Napoli, Italy \\ acanfo18@posteitaliane.it \\ Renato Aurigemma \\ Euro.Soft \\ Trav. Italia 68 \\ Pozzuoli, Italy
r.aurigemma@eurosoftsrl.eu s.muto@eurosoftsrl.eu
}

\author{
Cosimo Birtolo \\ Poste Italiane \\ Information Technology \\ P.zza Matteotti 3, Napoli, Italy \\ birtoloc@posteitaliane.it \\ Massimiliano Tafuto \\ Protom Group \\ via V.S.Maria del Pianto \\ Napoli, Italy \\ massimiliano.tafuto \\ @protomgroup.com
}

\begin{abstract}
The growing importance of ubiquitous and context-aware computing has led to an increasing interest in location-based mobile application. In this paper, we are going to describe the concept of indoor localization, as well as a real-world application to a museum. Indoor localization is a key feature of FIBAC, a research project aimed at creating a system prototype able to set up customized visit routes in museums and art galleries, thanks to a Mobile App which will locate the visitor within the environment. For these reasons in this paper we are going to experiment fingerprint and trilateration algorithms by means of two real-case studies. Starting from the analysis of the correct room localization rate and the localization error of different scenarios we describe the adopted approach within a real museum environment (i.e., National Archeological Museum of Naples). Experimentation has proven the feasibility of localization-based services in this kind of context.
\end{abstract}

\section{Categories and Subject Descriptors}

H.3.4 [Information Storage and Retrieval]: Systems and Software; C.2.4 [Computer-Comunication Networks]: Distributed Systems

\section{General Terms}

Algorithms, Measurement, Experimentation, Human Factors

\section{INTRODUCTION}

Detecting the position of the user is a key feature in most of mobile applications. Many services provide useful informa-

Permission to make digital or hard copies of all or part of this work for personal or classroom use is granted without fee provided that copies are not made or distributed for profit or commercial advantage and that copies bear this notice and the full citation on the first page. To copy otherwise, to republish, to post on servers or to redistribute to lists, requires prior specific permission and/or a fee.

I-LOCATE 2014, December 02-04, London, Great Britain

Copyright (c) 2014 ICST 978-1-63190-039-6

DOI 10.4108/icst.mobiquitous.2014.258032 tion related to the position of the user. The Global Positioning System (GPS) covers most of the surface of the planet and the cost of GPS chipsets is continually decreasing, making their integration into mobile devices easier.

However, GPS is unreliable in indoor environments, since walls (e.g. offices, hospitals, museums) hinder the signals transmitted by GPS satellites. Many recent research works deal with the persisting issues of indoor localization. First of all, since two rooms are generally closer than two streets, indoor localization requires a higher localization accuracy. A 10-meter localization error in an outdoor environment is not as important as it is in a building. Secondly, indoor localization often needs a calibration phase which can be expensive because of the high cost of the technologies required. Different research works are trying to address these issues as indoor localization becomes more and more widespread in several domains.

Localization Based Services (LBS) are crucial in the tourism domain and context-aware information could make the difference between relevant information and redundant news. In particular, we have focused on the environment of a museum, where context-aware information can characterize users visits, thanks to a customized application specifically designed for mobile devices, which will provide the visitor with a personal guide within an unknown environment. Indeed, the identification of the room visited by a user can enable content related to the room itself and its artworks.

The purpose of this paper consists in analyzing the state-ofthe-art of indoor localization, experimenting this technique in the context of a museum. In particular, we have considered two real-case studies: (i) an office and (ii) the National Archeological Museum of Naples, where visitors can admire the artworks on the base of their arrangement on the walls of each room. The first case study was investigated in order to compare existing localization techniques by simulating typical visitors' routes, while the second one (a real museum) was considered in order to validate the selected approach. This latter scenario led to some research questions about the success of the localization rate in a museum, which is the 
main domain of application of the FIBAC project (Fruizione Innovativa dei Beni Artistici e Culturali - Innovative Fruition of Cultural Heritage Assets). FIBAC is currently an ongoing project (it is expected to end in 2015) conducted by Poste Italiane, University of Salerno and some small and medium Italian enterprises, such as Protom Group, Space, Meta, Nexsoft, and Lit Com. The project is focused on the definition of a system for the dynamic generation of customized routes in museums and art galleries in order to deliver customized and context-aware information which will allow a better understanding of the artworks, on the base of a narrative structure. Out of all the different objectives this project aims at, this research work takes into account the specific aspects of indoor localization in museums.

The paper has been arranged as follows: Section 2 provides a brief overview about the context of the museum and indoor localization systems; Section 3 describes related works and localization technologies; Section 4 and 5 present the experimentation carried out; Section 6 describes the real-world application and Section 7 outlines conclusions and orientations for future research.

\section{MUSEUM INDOOR LOCALIZATION}

Information technology has changed the relationship between visitor and museum [4]. In the past, museum communication was usually delivered through consistent informative panels and brochures. Today, new media and social channels allow a shared dialogue between users and museums which actively involves both of these subjects [5]. Recent national studies outlined three different scenarios: i) the Pragmatic $\mathrm{Mu}-$ seum; ii) the Matrioska Museum and iii) the Playable $\mathrm{Mu}-$ seum. Each one of them emphasizes a specific aspect of the museum experience, such as, respectively: i) customization; ii) in-depth analysis; iii) the possibility of playing games. More specifically, "Pragmatic Museum" is focused on the customization of both the duration and the content of the visit, according to the organization of the user. This scenario requires Web, e-Collaboration and mobile localization technologies. "Matrioska Museum" provides content and customization on the base of the specific requests of the user. This scenario requires smartphones and connectivity, RFID and GPS. Finally, "Playable Museum", where education goes hand in hand with entertainment. This scenario requires interactive technologies and systems, in order to involve communities in virtual environments. The common aspect of these three scenarios is the mobile device. In 2007 , Marota [8] identified 6 different usage patterns for a mobile device during a visit to a museum:

1. Informative: the device integrates pictures, videos and other typical functions of audio-guides into the approach of a paper travel guide, so as to direct users' attention more effectively. The inclusion of accessible contents breaks the sequentiality arranged by the curator and expands the physical space expands the physical space of the museum, leading it through virtual channels to potentially borderless areas.

2. Prosthesis: the device is designed as a tool which allows the manipulation of digital experiences. Marota [8] states that remodeling a sculpture or changing the colors of a painting offers visitors a more practical contact with artworks, which takes concrete shape when information is delivered through procedural learning schemes.

3. Consultant/Prompter: the device works like an expert guide who suggests artworks in the exhibition which can potentially interest the user (according to specific criteria such as the author, the artistic movement, iconography, and so on). It reacts to inputs from the user, processing them so as to offer a new interpretation of the wider idea of museum. During the visit, it can also suggest merchandise related to the preferences of the user, exploiting the emotional effects artworks produce on him/her.

4. Navigator: thanks to the use of multiple technologies, the device provides spatial orientation through the halls of the museum, showing the visitor artworks, services and other areas of interest.

5. Communicator: thanks to audio message functions, the device enables communication within the museum and during the visit, so that visitors can share their experience and the museum administration can send them relevant information and promotional messages.

6. Witness: the device works as a private or public memory of the visit experience. In the former case, visitors can acquire the information delivered during previous visits and stored in third devices, just by accessing them through their own device, so as to extend the visit experience itself and foster a sense of belonging to the museum as an institution. In the latter case, the museum becomes a database of collective knowledge through the collection of assessments, comments and opinions expressed by users.

The thematic roles proposed for the device have been designed according to the habits, preferences and needs of both visitors and operators. By comparing the results of 4,549 inquiries carried out during two different surveys (in 1999 and in 2011), a recent study [13] has shown changes in the taste and needs of Italian museum visitors over the past 12 years. The data show that in Italy the majority of visitors is female (56\%), Italian (58\%) and graduate (64\%). Only in the $13-14 \%$ of cases visitors are young (aged between 15 and 24) or over 65 , and they usually go to the museum with their families and friends. Moreover, data show that $60 \%$ of visitors have been informed about the exhibition proposed by the museum ( $67 \%$ of which through the Internet). Services offered by museums do not fully meet visitors' expectations, as $33 \%$ of them experience difficulties in finding the specific information they are looking for. Users ask for thorough information about the whole exhibition, its historical and geographical background, as well as specific details about single artworks. Visitors' interest is mainly focused on living a sort of cognitive, aesthetic and hedonic experience. They are willing to meet knowledgeable and experienced guides, and $40 \%$ of them turns out to be favorable to the use of technological tools (such as tablets and smartphones) during the visit. The interest in the use of innovative devices is inversely proportional to the age of the visitors interviewed. These considerations has been confirmed by several studies 
at international level. Indeed, the Smithsonian Institution ${ }^{1}$ affirms that technology in an art museum enhances the visit experience, while the Sackler Gallery states that "four out of five visitors believe that both videos and interactive devices are useful in art museums" ${ }^{2}$ According to Goldman's work [3], visitors are favorable to the use of new technologies and mobile devices. A museum visit is a dynamic experience, and the study carried out by D. Wessel and E. Mayr [15] has shown that such an experience can be improved by the use of technology in response to the need for a small, lightweight and transportable device designed to give access to the services offered by the museum. These features make the device suitable to individual users, but not for the needs of groups of visitors. On the basis of these considerations, mobile devices can provide highly customized information as they can be adapted to the characteristics of the visitor. The possibility to use their own mobile phones allows visitors to enjoy the whole visit, without wasting any time on learning how to use a new device, so as to avoid cognitive overload and reduce the cost of technology for the museum [1]. Moreover, LBS within museums are enriched using mobile device in pre-visit and post-visit phases, allowing visitors to look for additional information through the web. In particular, pre-visit inquiries will enable visitors to identify artworks and exhibitions which will interest them the most, giving a greater focus to their visit, while post-visit inquiries will allow them to deepen their knowledge by acquiring further information and linked references.

IBM developed a particularly interesting app related to Italian museums, which is available (with some differences) both for Android ad iOS. The device detects the position of each visitor and suggests a "smART" tour with routes leading to the different artworks. Moreover, the app allows the visitor to photograph the QR code inside the MAXXI museum (National Museum of XXI Century Arts) and acquire information about the artworks located in each room. Similarly, the American Museum of Natural History aims at ensuring a better experience for visitors, both on-site and on-line, by facilitating the navigation, by providing an application that encourages to repeat the visits, and by improving the information without affecting the exhibitions. This app was developed for the iOS operating system, and studies have proven that Wi-fi triangulation allows an identification of the location inside the museum accurate enough to detect the exhibition hall where the visitor is.

\section{LOCALIZATION TECHNOLOGIES}

Several relevant projects concern issues related to localization within museums and, more generally speaking, indoor localization. The i-Locate project is focused on three scenarios: (i) Guidance of patients through a health care path, (ii) Smart tracking and asset management for lean asset management models, and (iii) Guiding visitors to reach specific indoor locations ${ }^{3}$. In particular, the third scenario implies location-based e-gov services, and the museum experience is an example of their application. In this domain, the PEACH

\footnotetext{
${ }^{1}$ http://www.si.edu/

${ }^{2}$ Institutional Studies Office, America Meets Asia: A Report based on the 1995-1995 Freer Gallery of Art and Arthur M. Sackler Gallery Visitor Study (Washington, D.C.: Smithsonian Institution, 1995).

${ }^{3}$ http://www.i-locate.eu/
}

project (Personal Experience with Active Cultural Heritage) has produced a multimedia platform able to localize the visitor in the museum rooms. InLite [11] is an indoor navigation system that uses pseudo satellites to locate subjects in big multi-level buildings.

\subsection{Methodologies for indoor localization}

In 2010, Jorge Torres-Solis et al. [14] classified some selected papers, related to the indoor localization context, into five categories (i.e., radio frequency, photonic, sonic waves, mechanical, other) according to the physical quantities measured (see Fig. 1).

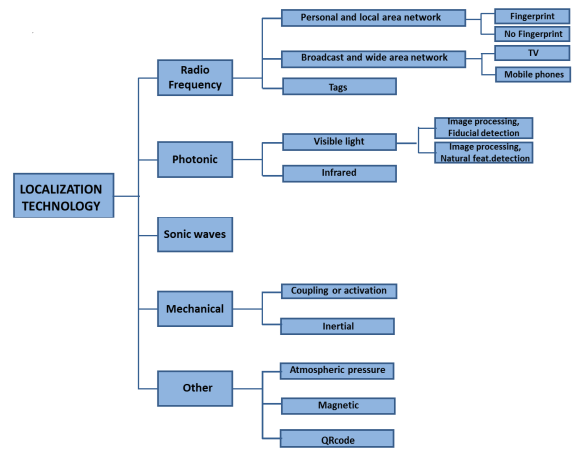

Figure 1: Taxonomy of indoor localization technologies $[14]$.

Fig.2 summarizes the technological details, the localization technologies and the metrics Torris-Solis adopted for the above-mentioned classification. The acronyms used stand for: TR for triangulation, PR for proximity, SA for scene analysis, DR for dead reckoning; $\mathrm{T}$ time, $\mathrm{D}$ direction, I intensity, and $\mathrm{O}$ other.

An in-depth analysis of such technologies has shown that the systems based on WLAN, Bluetooth, ZigBee and UWB technologies are characterized by simple and cheap architecture. On the other hand, these technologies are able to produce just a rough localization (as they're characterized by a meter-level accuracy), they often require an off-line work-phase in order to track the signal and they're very sensitive to noises and environmental changes. n addition to the need for signal mapping, LTE and WiMax-based systems also suffer from a lack of infrastructures. RFID and NFC-based systems offer a centimeter-level accuracy but have a limited operative range. Setting them up is not expensive, since the RFID TAG are very cheap and the NFC technology is installed on a lot of smartphones. Systems using the Pattern Recognition offer a good accuracy (less than a meter) and are able to direct the user. However, their performances are highly affected by light conditions. Systems based on Dead Reckoning are very resistant to environmental changes, and provide a continuous upgrade of the position of the user. Unfortunately, these systems must be constantly calibrated due to their tendency to accumulate error. In 2003, radio waves were taken into account in order to locate the position of the user through specific algorithms. Wi-fi radio waves had been initially used, but they were then replaced by UWB and Bluetooth. In 2008, several studies examined ZigBee-based localization systems, as 


\begin{tabular}{|c|c|c|c|c|c|c|c|c|c|}
\hline \multirow{2}{*}{$\begin{array}{c}\text { Main } \\
\text { Technology }\end{array}$} & \multirow{2}{*}{ Details } & \multicolumn{4}{|c|}{$\begin{array}{l}\text { Localization } \\
\text { technique }\end{array}$} & \multicolumn{4}{|c|}{ Metrics } \\
\hline & & TR & PR & SA & DR & $\mathbf{T}$ & D & I & O \\
\hline \multirow{10}{*}{$\begin{array}{l}\text { Radio } \\
\text { Frequency }\end{array}$} & WLAN & $\checkmark$ & $\checkmark$ & $\checkmark$ & & $\checkmark$ & $\checkmark$ & $\checkmark$ & \\
\hline & Bluetooth & $\checkmark$ & $\checkmark$ & $\checkmark$ & & $\checkmark$ & $\checkmark$ & $\checkmark$ & \\
\hline & ZigBee & $\checkmark$ & $\checkmark$ & $\checkmark$ & & $\checkmark$ & $\checkmark$ & $\checkmark$ & \\
\hline & UWB & $\checkmark$ & $\checkmark$ & $\checkmark$ & & $\checkmark$ & $\checkmark$ & $\checkmark$ & \\
\hline & LTE & $\checkmark$ & & $\checkmark$ & & $\checkmark$ & $\checkmark$ & $\checkmark$ & \\
\hline & WiMax & $\checkmark$ & & $\checkmark$ & & $\checkmark$ & $\checkmark$ & $\checkmark$ & \\
\hline & Pseudolite & $\checkmark$ & $\checkmark$ & $\checkmark$ & & $\checkmark$ & $\checkmark$ & $\checkmark$ & \\
\hline & FM & $\checkmark$ & & $\checkmark$ & & $\checkmark$ & $\checkmark$ & $\checkmark$ & \\
\hline & RFID & & $\checkmark$ & $\checkmark$ & & $\checkmark$ & $\checkmark$ & $\checkmark$ & \\
\hline & NFC & & $\checkmark$ & $\checkmark$ & & & & & $\checkmark$ \\
\hline \multirow{2}{*}{ Photonic } & Pattern R. & & & $\checkmark$ & & & & & $\checkmark$ \\
\hline & Infrared & $\checkmark$ & $\checkmark$ & $\checkmark$ & & & $\checkmark$ & & \\
\hline Sonic & Ultrasound & $\checkmark$ & & & & $\checkmark$ & $\checkmark$ & $\checkmark$ & \\
\hline \multirow[b]{2}{*}{ Mechanical } & Sensor & & $\checkmark$ & & & & & & $\checkmark$ \\
\hline & $\begin{array}{l}\text { Dead } \\
\text { Reckoning }\end{array}$ & & $\checkmark$ & & $\checkmark$ & & & & $\checkmark$ \\
\hline Other & QR-Code & & $\checkmark$ & & & & & & $\checkmark$ \\
\hline
\end{tabular}

Figure 2: Taxonomy of methodologies/technologies for indoor localization.

well as the sensors embedded in the last generation of smartphones, that are able to realize dead reckoning techniques. Although all these kinds of techniques are very interesting, the experimentation described in this paper will be focused on radio-frequency-based technologies, which can be classified as follows:(i) Personal Area Network (PAN) and Local Area Network (LAN): IEEE 802.11 (WLAN); IEEE 802.16 (WiMax); Bluetooth; ZigBee; Ultra-Wideband (UWB); (ii) Broadcast and Wide Area Network: mainly WAN networks; and (iii) Tag (RFID, NFC). Thanks to PAN and LAN, mobile device localization can be carried out through one of the solutions proposed, or even through a hybrid solution which combines together two or more of the above-mentioned techniques. Fingerprint or Triangulation algorithms are usually used in order to locate a device. The most common metrics are those based on time, direction, and intensity of the radio signal. The following paragraph show an overview of the PAN and LAN technologies for indoor localization, highlighting the relevant scientific studies.

\subsection{Algorithms}

Localization algorithms can be classified into two main categories: (i) Geometrical: these kinds of algorithms locate the position using geometrical principles and rules; Scene analysis (Fingerprint): these kinds of algorithms locate the position using a prior mapping of the environment. Moreover, there are hybrid algorithms that locate the position by combining several techniques and exploiting the difference between each technique's error rate. The device used to localize will be called MS (Mobile Station) and the localization stations will be called BS (Base Station).

\subsubsection{Geometrical Algorithms}

Lateration is a technique that calculates the position of a device by measuring the distance between the MS and a set of known and fixed points, which are the BS's. In trilateration algorithm, if the position of the BS is known, as well as its distance from the device (MS), the position of this latter will coincide with one of the endless points located on the circumference whose center matches with the position of the $\mathrm{BS}$ and whose radius coincides with its distance from the device. If this operation is repeated for each one of the three BS's, three different circumferences will be generated, and the position of the device will coincide with the point standing simultaneously on all of the three circles [4]. Basically, in order to determine the position of a MS it is necessary: (i) to detect the wi-fi signal, (ii) to identify BS, and (iii) to deduce its position by interpreting the intensity of the signal in terms of distance.

The hyperbolic lateration [4] is based upon the calculation of the of the difference in distance between the MS and 2 BS's. The use of measurement techniques in order to estimate the difference between the two distinct estimated times of arrival will make it possible to put some limits to the potential position of the MS within the space considered. The MS position will be located on a hyperbolic line, with the two BS's as focus points. Three BS's generate three hyperboles (even though two are enough), and the MS position will coincide with their intersection. The triangulation algorithm is based upon the direction of the MS arrival signal estimation made by the BS. In this algorithm the position of the MS can be determined by the intersection of several couples of angles direction lines, each joining the single BSs to the MS. An advantage is that this algorithm does not need any synchronization time. On the other hand, the hardware needed for its implementation is very complex and the estimation of the position get worse in relation with the distance between MS and BS since the signal is subject to multiple reflections. Finally, the proximity algorithm [7] is based upon the creation of a thick grid of antennas (BS), each one of them in a known position. When a device (MS) is detected by an antenna, the position of the device will coincide with the position of the antenna. When the device detects the signal of multiple antennas, the MS position will coincide with the position of the antenna transmitting the strongest signal, or with a position equal to the weighted average of the different measurements carried out. This system is mainly implemented by infrared and RFID technology.

\subsubsection{Fingerprint}

Fingerprint algorithms, also named "Scene Analysis", are mainly used for wireless localization, thus it is possibile to use them in different contexts as well, such as localization through video cameras or passive infrared sensors. Scene Analysis algorithms consist of two different phases [10]:

- Offline or training's, when the radio map, when the radio map, including all the signal levels for different space points, is created

- Online, in which the position of the device is determined using localization algorithms based upon the matching of the measurements acquired. 
At the end of the first phase, the device, that detects the signal, compares it with the measurements obtained during the training phase, estimating the device position on the base of the similarity between the actual measurement and the one recorded during the training phase. The similarity between the measurements can be calculated in several ways, but the Euclidean distance is often used. For instance, if we consider the measurements of $N$ access points related to the two phases, $\left(S_{1}, S_{2}, \ldots, S_{N}\right)$ for the offline phase and $\left(R_{1}, R_{2}, \ldots, R_{N}\right)$ for the online phase, the Euclidean distance is calculated as follows:

$$
E=\sqrt{\left(S_{1}-R_{1}\right)^{2}+\left(S_{2}-R_{2}\right)^{2}+\cdots+\left(S_{N}-R_{N}\right)^{2}}
$$

Roxin et al. [10] classified two different types of algorithm to use during the online phase: (i) algorithms with deterministic techniques, such as Nearest Neighbour in Signal Space (NNSS), Nearest Neighbour in Signal Space-Average (NNSS-AVG), Approximate Point-In-Triangulation (APIT); (ii) algorithms with probabilistic techniques ((such as the conditioned probability principle and Bayer's theorem [7]).

\subsection{Remarks}

Inquiries carried out on research projects, business solutions and smartphone applications have revealed that, at the moment, only a few solutions for indoor localization are available, as well as that technological solutions fully integrated into mobile devices are to be preferred for museums. Other hardware solutions are still too expensive. Furthermore, the Dead Reckoning technique, although integrated into devices, still suffers from a severe limit related to error accumulation. Although the importance of topographic information for a visit to a museum has been made widely clear, out of all the apps considered, only a few provide location-based services at the moment. Wi-fi technology is usually preferred for the rare cases when a tracking service is provided. Moreover, it's worth pointing out the fact that, although it is always preferable to use localization systems as precise as possible, a less accurate localization technique still can be effective in order to provide useful information about artworks.

\section{MATERIALS AND METHODS}

The experiments described in this paper were conducted in a typical office environment consisting of open space rooms (see Fig.3) and in a real museum environment, i.e., MANN (Museo Archeologico Nazionale di Napoli, National Archeological Museum of Naples) as depicted in Fig.4. Experiments were conducted by a human operator carrying a smartphone HTC Desire HD and Samsung Galaxy Tab2 with Android OS. Three wireless devices (i.e., D-Link DIR-300 called "Dlink", Shintek Wireless G 54 Mbps called "Shintek" and Cisco AIRAP1121G-E-K9 called "Cisco") were placed at the indicated locations to cover the entire area in both experiments. In the office environment the Access Points (APs) or Base Stations (BSs) were placed at various heights from the ground (DLink: $1.72 \mathrm{~m}$, Cisco: $2.33 \mathrm{~m}$; Shintek: $2.15 \mathrm{~m}$ ). In the museum environment the devices were placed at a lower heights with respect to the previous ones for technical issues.

The hardware selection for experiment was heterogeneous and low cost, which are a worst-case condition and represent a generic network infrastructure not specifically created for the purpose of indoor localization.

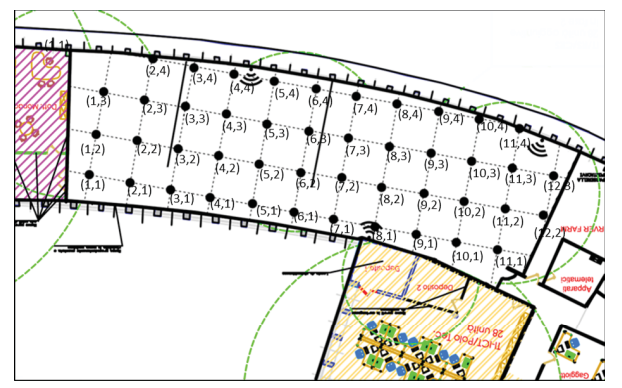

Figure 3: Office Environment showing the location of the three wireless Access Points and the grid.

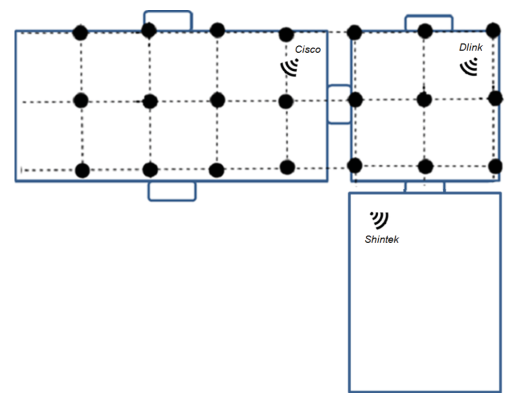

Figure 4: Museum Environment showing the position of Access Points and the grid.

In Section 5, we are going to prove the variability of AP's signal power. Generally speaking, it should be highlighted that Cisco AP presents an inefficiency exceeding of about $25 \%$. Other APs do not show signal loss, but they have extreme variability in signal power (14 e $15 \mathrm{dBm})$. It is one of the worst cases, compared with situation of stable signal power[12].

\subsection{Procedure}

As preliminarily described in Section 3, a typical system of indoor localization needs two main steps, an offline phase, in order to build a database of fingerprints of signals reached from every access point, and an online phase, in which the signal is acquired instantly.

First of all, a set of Reference Points (RPs) was selected, and a Mobile Station (MS) was positioned in every RP in order to measure the signal of different APs. Taking the hardware used and the literature in this field in consideration, it was regarded as appropriate to test three different types of mapping: (i) with a single orientation, (ii) with four orientations, and (iii) with four filtered orientations. In the case of single-orientation mapping for each one the 45 RPs selected, the strength of the signals taken by each AP was considered directly, without any alteration, and the database was filled with the arithmetic mean of 100 scans. In the case of fourorientation mapping, instead, the antenna directivity of the mobile was considered, because there could be a different sensitiveness depending on the orientation between mobile and AP, as described in [2] and [9]. So for each RP 100 scans were taken at $0^{\circ}, 90^{\circ}, 180^{\circ}$ and $270^{\circ}$. For these orientations the arithmetic mean was calculated and the database was filled with the new arithmetic mean of these values. Be- 
cause of the instability of signals, caused by the commercial hardware adopted, some filtering techniques were used in order to remove erroneous spikes. So, for every orientation, 100 scans were taken as usual, and the mean values $(\mu)$, the variance and the standard deviation $(\sigma)$ were calculated. In particular, the mean of the values belonging to the interval $[\mu-\sigma, \mu+\sigma]$ was taken in consideration.

During the online phase, which typically consists in a simple comparison between the signal acquired from the mobile at runtime and the set of values stored in the database, two different typologies of acquisition were planned: (i) 100 scans, from which the mean value to compare with those of the mapping was calculated, (ii) 100 filtered scans, with a selection based on the comparison with the standard deviation, such as it was planned for the offline step.

In order to validate the algorithms tested in the online step, we planned to perform two different acquisitions of signals for the comparison, a static one and a dynamic one. In the former modality the device was stationary during the acquisition performed for the online phase. In the latter one, instead, the localization was performed while the user was moving in the experimentation area.

\subsection{Metrics}

In order to measure the signal intensity for localization purposes, the RSSI (Received Signal Strength Indicator) was taken in consideration. In an empty space, the RSSI value varies depending on the square of the distance $\mathrm{d}$ between the transmitter and the receiver. Saying $P_{r}(d)$ stands for the received power depending on the distance $d$, we have:

$$
P_{r}(d)=\frac{P_{t} G_{t} G_{r} \lambda^{2}}{(4 \pi)^{2} d^{2}}
$$

where $P_{t}$ is the transmitter power, $G_{t}$ is the transmitter antenna gain, $G_{r}$ is the receiver antenna gain, and $\lambda$ is the wavelength of the transmitted signal expressed in meters. The propagation in an empty space is just an idealization, since the signal propagation is affected by reflection, diffraction and scattering phenomena. Therefore, in order to calculate the distance and carry out the localization it is necessary to apply adjusting factors. RSSI decays with the square of the distance as a function of the space it crosses [6], so that the distance $d$ for the trilateration algorithm is evaluated as follows:

$$
d=10^{\frac{10 \log \left(P_{0}\right)-10 \log \left(P_{r}\right)}{10 \alpha}}
$$

where $P_{0}$ indicates the signal intensity at a distance of one meter and $\alpha$ is a coefficient of attenuation typical of the environment. The values of $P_{0}$ were obtained by placing the $\mathrm{MS}$ at a distance of one meter from each AP, and performing 100 scans. The mean RSSI value was used to determine the three $P_{0}: 42.33 \mathrm{~dB}, 49.25 \mathrm{~dB}$, and $41.25 \mathrm{~dB}$ respectively for Cisco, Shintek and D-Link AP. Considering that a museum is often situated in a historic building, where the walls between rooms are very thick and it is difficult for the signal to cross them, or in large areas, where the signal does not meet any hindrances, an empirical evaluation of $\alpha$ was selected.

\section{EXPERIMENTATION}

In this section we are going to describe the different experimentation performed. First of all, we are going to analyze the way signals'power differs as different mobile devices are used (both smartphones and tablets). Secondly, we are going to test fingerprint and trilateriation algorithms described in Section 3. Then, we are going to examine the results in order to define pros and cons related to different options for a real museum environment.

\subsection{Experimenting signal strength detected by two mobile devices}

For the correct execution and interpretation of experiment results, we need to estimate the signal strength of access points. Fig. 5 shows a plot of the signal strength recorded for the three APs, i.e., Shintek (see Fig.5(a)), Cisco (see Fig.5(b)) and Dlink (see Fig.5(c)). Signal strength was recorded by smartphone and tablet in 100 consecutive scans per second in the same point of experimentation area. These measurements were carried out twice. The second measurement confirmed that the signal strength recorded by the access point was very variable. In other words, data analysis did not show any predictable behavior and so it is not possible to take countermeasures to limit the influence of this uncertainty in the following tests.

Another important consideration is about the way smartphones and tablets are held. Antennas severely suffer from the hindering effect caused by users' hands. This problem seems not so important when using a tablet, but it becomes more relevant with mobiles, in which the antenna is located at the top of the device. When the device is held in a vertical position, the problem is almost negligible, but when the device is held horizontally, there is a substantial decrease of the signal which can adversely affect the efficiency of the algorithm. The different sensitivity of the hardware and the way the device is held affect the performance of localization systems, producing a considerable decay in the quality of the localization. However, this is a problem scientific literature has rarely dealt with, and there is no single solution for it.

\subsection{Fingerprint Algorithm Experimentation}

In order to investigate the accuracy of the fingerprint technique, an office environment was selected (see Fig.3) as well as $k-n n$ algorithm, with a $k$ value ranging from 1 to 7 , according to $\mathrm{Li}$ et al. [6]. Therefore, three scenarios were tested, consisting in an online step with 100 scans and an offline one with different types of mapping, as explained in Section 4. For each test the localization process was carried out in each RP and for every value of $k$. Once defined the correct position (PR) and acquired the estimated position (PS), it is possible to calculate the localization error $\left(e_{r}\right)$, as follows: $e_{r}=\left|P_{R}-P_{S}\right|$

In the first test (single orientation mapping) the $\mathrm{k}$ with most instances and with minimum error is $k=6$, in the second test (four-orientations mapping) it is $k=5$, while in the third one (four-filtered-orientation mapping) it is $k=4$ (even if for $k=5$ a similar behavior was recorded).

Furthermore, it has been noticed that the RPs with the highest error rates are the one on the outer sides of the room, typically affected by structural defects such as refraction and multipath fading. Fig. 6 compares the results obtained for each algorithm in every RP. The graph also highlights the 


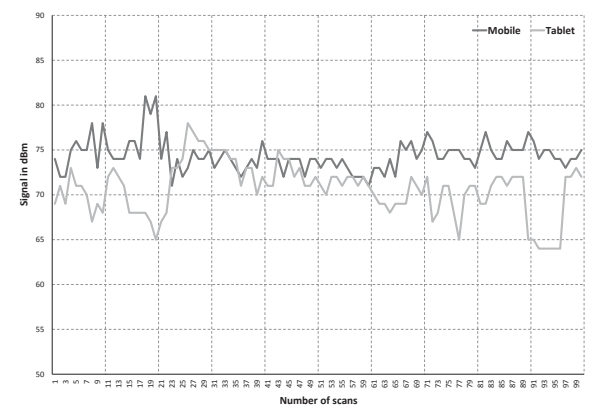

(a) Shintek

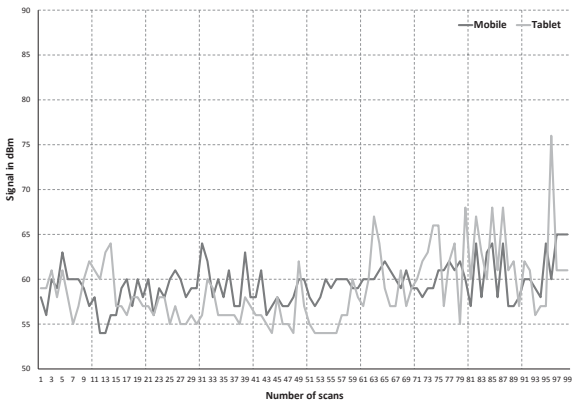

(b) Cisco

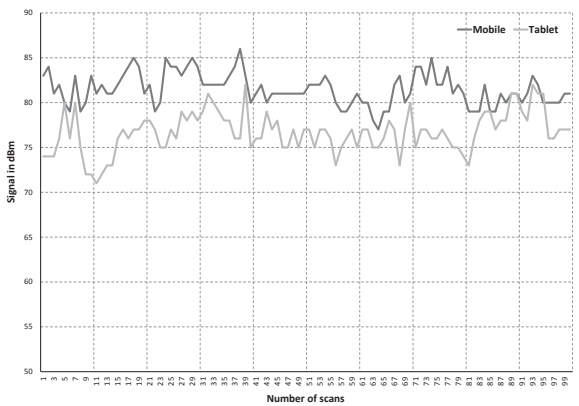

(c) Dlink

Figure 5: Variation of signal strength detected by smartphone (black) and tablet (grey).

peaks of errors repeated in the same RPs.

The Correct Localization Rate (CLR) is the ratio between the number of instances correctly located and the total number of occurrences. CLR can be defined at a whole-room level (correct room localization rate) or at a specified level within the room (i.e., 3, 4, 5, and 6 meters). In Table 1 , results show a success rate within the room for all of the three configurations.

In order to thoroughly analyze these findings, in Fig. 7

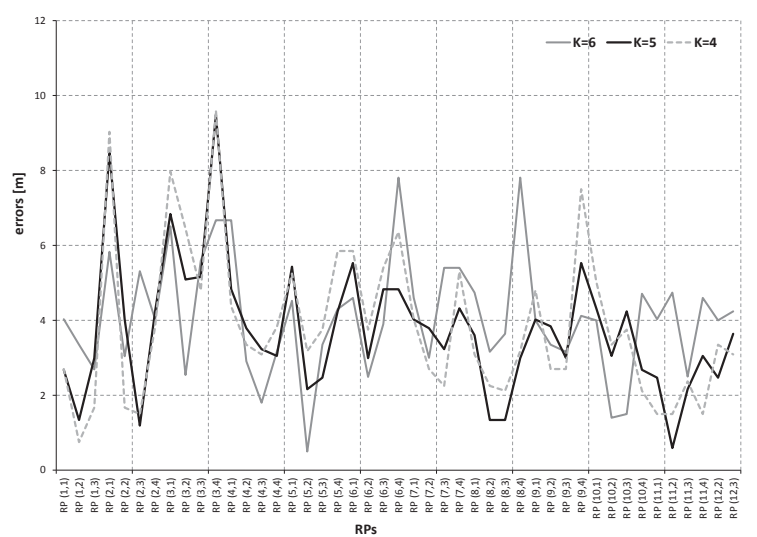

Figure 6: Comparison between algorithm selected in every test.

Table 1: Fingerprint Algorithm: CLR as a function of $K$ and localization threshold

\begin{tabular}{|c|c|c|c|c|c|}
\hline $\mathrm{K}$ & Room & $3 \mathrm{~m}$ & $4 \mathrm{~m}$ & $5 \mathrm{~m}$ & $6 \mathrm{~m}$ \\
\hline 6 & $100 \%$ & $22 \%$ & $49 \%$ & $78 \%$ & 89 \\
\hline 5 & $100 \%$ & $36 \%$ & $58 \%$ & $82 \%$ & 93 \\
\hline 4 & $100 \%$ & $36 \%$ & $64 \%$ & $73 \%$ & 87 \\
\hline
\end{tabular}

we have plotted the number of occurrences which have a localization error equal or lower than the one specified on the x-axis. We can state that, for errors smaller than 5 meters, the best algorithm is $k=4$, between 5 and 7 meters it is $k=5$ and for errors greater than 7 meters it is $k=6$.

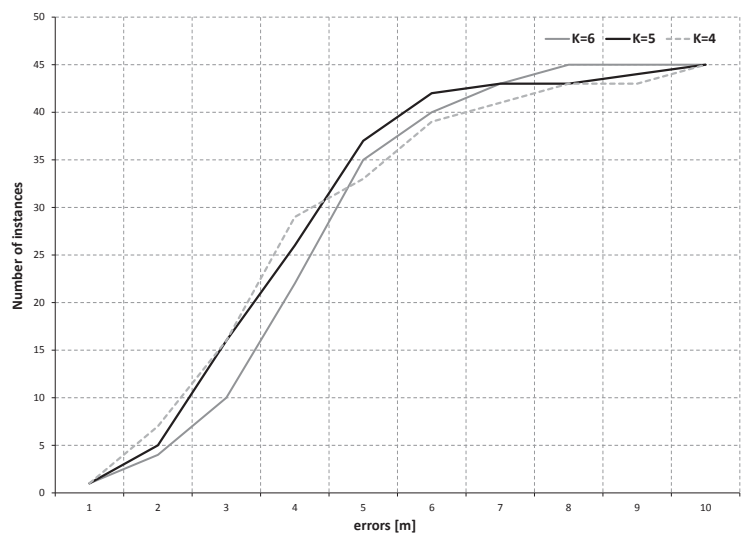

Figure 7: Comparison between errors at varying $k$ values.

In the FIBAC project, whose aim is to localize the user in the room, $k=5$ was selected. In order to optimize the waiting time, the test was repeated several times using different series of scans. It has been proven that a reduction of the number of scans from 100 to 10 makes the algorithm ten times faster without affecting significantly its results.

Once selected $k$ value was selected, the dynamic modality was tested on two different routes. In the first one, about 90meter long, for most of the time the user had to walk close 


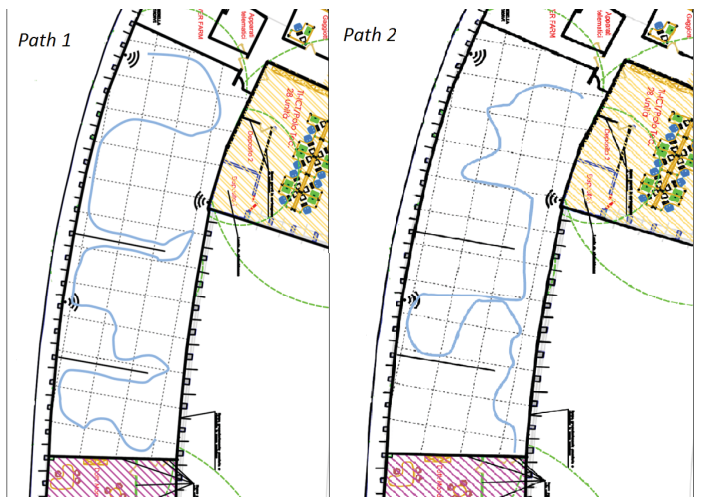

Figure 8: Routes simulated for the dynamic modality.

Table 2: Fingerprint Algorithm: Mean and max error at varying route and number of scans.

\begin{tabular}{|c|c|c|c|}
\hline Path & Scans & Mean error & Max error \\
\hline 1 & 5 & 3.35 & 7.58 \\
\hline 1 & 10 & 3.51 & 5.55 \\
\hline 2 & 5 & 2.58 & 4.36 \\
\hline 2 & 10 & 2.88 & 4.26 \\
\hline
\end{tabular}

Table 3: Fingerprint Algorithm: CLR at varying route and number of scans.

\begin{tabular}{cccccccc} 
& & & \multicolumn{5}{c}{ CLR } \\
Path & Scans & & Room & $\mathbf{3} \mathbf{~ m}$ & $\mathbf{4} \mathbf{~ m}$ & $\mathbf{5} \mathbf{~ m}$ & $\mathbf{6} \mathbf{~ m}$ \\
\cline { 1 - 4 } \cline { 5 - 8 } 1 & 5 & & $100 \%$ & $51 \%$ & $70 \%$ & $82 \%$ & $87 \%$ \\
1 & 10 & & $100 \%$ & $47 \%$ & $63 \%$ & $81 \%$ & $94 \%$ \\
2 & 5 & & $100 \%$ & $72 \%$ & $86 \%$ & $97 \%$ & $100 \%$ \\
2 & 10 & $100 \%$ & $68 \%$ & $87 \%$ & $100 \%$ & $100 \%$ \\
\hline
\end{tabular}

to the walls, while in the second one, which was a little bit shorter, the user had to cross the experimentation area (see Fig.8). The purpose of this choice was to simulate typical behaviors of cultural visitors, according to the way relevant literature describes them.

For each route the algorithm was launched three times, with 5 and 10 scans, in order to make experimentation results more reliable. The localization error rarely exceeds 5 meters when the user walks the first route, while it rarely exceeds 4 meters when he/she crosses the experimentation area. As shown in Tab. 2 and Tab.3, using 10 scans instead of 5 implies lower computation time and leads to better results, even when the visitor was walking close to the walls. In both cases, anyway, the room was correctly detected (see Tab.3).

\subsection{Trilateration Algorithm Experimentation}

Similarly to the fingerprint experimentation, trilateration was tested in an office environment (see Fig.3).

Such as in the case of fingerprint experimentation, experimentations were conducted according to two modes, a static
Table 4: Trilateration Algorithm: CLR without correction and with correction.

\begin{tabular}{|c|c|c|c|c|}
\hline Algorithm & $3 \mathrm{~m}$ & $4 \mathrm{~m}$ & $5 \mathrm{~m}$ & $6 \mathrm{~m}$ \\
\hline Trilat & $28.8 \%$ & $42.2 \%$ & $46.6 \%$ & $55.5 \%$ \\
\hline $\begin{array}{l}\text { Trilateration } \\
\text { with correction }\end{array}$ & $28.8 \%$ & $44.4 \%$ & $53.3 \%$ & $64.4 \%$ \\
\hline
\end{tabular}

one and a dynamic one.

In order to compare the data obtained with the two different techniques for the static mode, the Trilateration algorithm was applied to the RSSI values detected during the fingerprint experimentation. The test was repeated three times and the data obtained are presented in the following charts, where, for each point of the map, the error in estimating the exact position (in meters) is shown (grey bar). In case the estimated point is located outside the grid area, it is possible to apply correction mechanisms. Such mechanisms tend to replace the item with the one estimated on the edge of the area closest to the testing point itself. Fig.9 shows the error in meters with the correction technique (black bar). Using this simple correction, it is possible to reduce errors and improve significantly the algorithm.

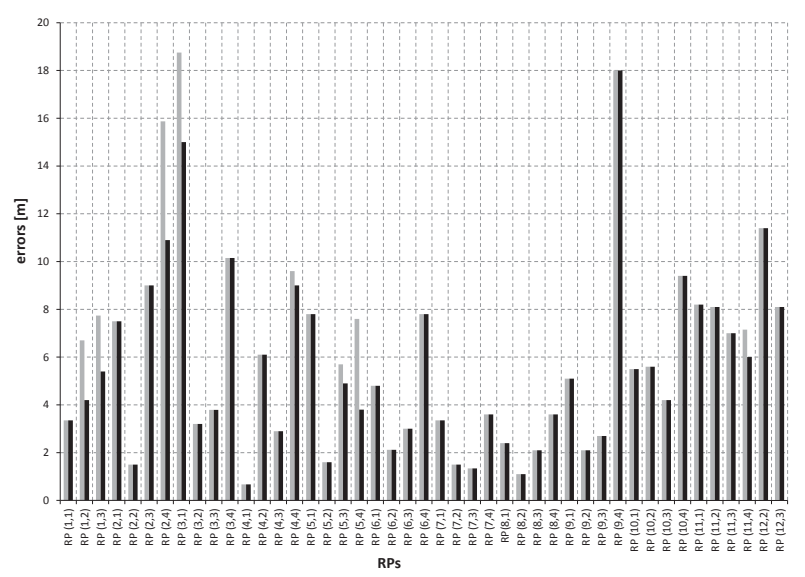

Figure 9: Error for each RP for trilateration (grey bar) and trilateration with error correction (black bar).

With this simple correction, the success rate is improved (compared to the case of standard trilateration), especially for severe errors (see Tab.4).

The second and third tests confirmed the results of the first. The results obtained with the static mode for trilateration show an error of 6 meters. So the performance of the algorithm was quite poor and success rate has never reached $70 \%$.

Experimentation with the dynamic mode was conducted in an office environment with the same routes and procedures used for the fingerprint experimentation (see Fig.8). Traditional trilateration and error correction techniques were taken in consideration, and the results obtained through the dynamic mode on the base of 10 scans (i.e., average CLR 
Table 5: Trilateration Algorithm: CLR at varying path.

\begin{tabular}{ccccccc}
\cline { 1 - 5 } Path & Correction & & $\mathbf{3} \mathbf{~ m}$ & $\mathbf{4} \mathbf{~ m}$ & $\mathbf{5} \mathbf{~ m}$ & $\mathbf{6} \mathbf{~ m}$ \\
\cline { 1 - 4 } \cline { 5 - 7 } 1 & No & & $15.53 \%$ & $34 \%$ & $54.13 \%$ & $60.63 \%$ \\
1 & Yes & & $15.53 \%$ & $37.92 \%$ & $56.20 \%$ & $65.53 \%$ \\
2 & No & & $26.67 \%$ & $31 \%$ & $38.33 \%$ & $50.83 \%$ \\
2 & Yes & & $26.67 \%$ & $35 \%$ & $46.67 \%$ & $65.83 \%$ \\
\hline
\end{tabular}

for a series of 3 tests) are shown in Tab.5.

Results in both routes show how trilateration leads to acceptable results for an error around 6 meters, while the percentages of success are very low for errors of 3-4 meters. Although the performances are similar for the two routes, the route 2 shows slightly lower errors due to the fact that it takes place in the innermost part of the area so that its points are less affected by errors due to multipath fading, reflection and refraction. On average, the error is about 5.5 meters, but it is nonetheless possible to find peaks that reach 12 meters. Although correction techniques can lead to better results, the maximum error is still quite high and it can produce a false detection of the room where the user is located.

\subsection{Comparing trilateration and fingerprint}

In order to compare the experimentation results obtained from the algorithms described, first of all, success rates in the dynamic mode were analyzed, since its characteristics are quite similar to the ones of a museum scenario.

Comparing Tab.3 with Tab.5, it is possible to state that fingerprint techniques show better performances than trilateration. There are two reasons for this: the fact that the signal is characterized by spikes and the hindering effect caused by objects which do not allow a correct translation of the signal intensity in distance. In addition, the translation of RSSI in distance still is a matter of discussion among researchers. On the other hand, fingerprint requires long times for the offline step: this can take up to 100 hours for a building of reasonable size. Moreover, this technique can suffer from changes in the environment.

In conclusion, it is possible to assert assert that trilateration algorithm shows better performances from the point of view of adaption to changes. For example, the addition or the removal of an AP in the trilateration algorithm would just require to add or delete a value stored in the database, while, in the fingerprint algorithm, it would require to calculate the offline step again. After all since the fingerprint algorithm is not affected by hindering effects, as they're included in the signal value, it produces better results in terms of precision and correctness. For these reasons, the fingerprint algorithm was selected as the ideal candidate for the validation phase in a real-world application at MANN museum.

\section{REAL-WORLD APPLICATION}

As mentioned before, for the validation phase a simulation in the National Archeological Museum of Naples was performed (see Fig.4) in order to prove the feasibility of the selected algorithm in a real museum. In particular, we selected two rooms dedicated to the "Tempio di Iside" on the first floor of the museum, as shown in Fig.10.

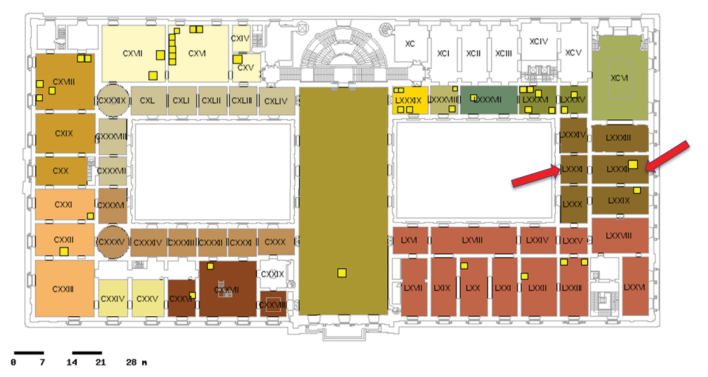

Figure 10: Isis' Temple: Rooms used for the experimentation.

The algorithm selected, as mentioned before, is fingerprint with $k$ values ranging from 1 to 7 , described in previous sections. For validation purposes, obviously, we used the same hardware of the experimentation inside the office and, in order to compare the results with those obtained in other experimentations, also the same mapping, with a $3 \times 3$ grid, which produced, in this case, a result of 21 RPs. Unfortunately, it was not possible to install the APs at exactly the same height, but we placed them on mobile supports near the electrical sockets available. Inside the museum, as a result of preliminary simulations, an offline step with a four-orientation mapping was planned. The online step, instead, was simulated in a dynamic mode (along the route shown in Fig.11) using 10 scans.

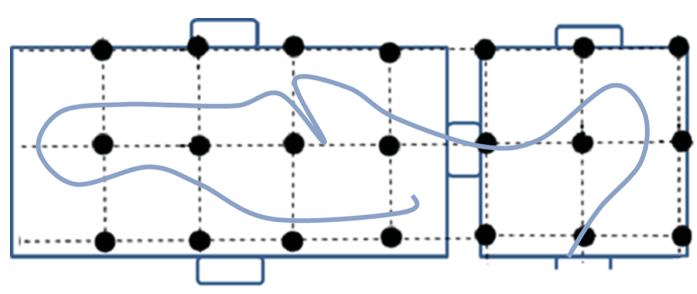

Figure 11: Isis' Temple: Visitor's route.

In this phase, we also tested the $k-n n$ algorithm with $k$ ranging from 1 to 7 . Fig.12 shows the number of times every algorithm produces the specified errors in meters. In this case, as in the office environment, the best behavior coincides with $k=5$, in which we find a mean error smaller than 6 meters as well as the smallest maximum error.

In MANN experimentation we detected a mean error equal to $2.88 \mathrm{~m}$ (maximum error is $5.99 \mathrm{~m}$ ), a correct room localization rate equals to $96 \%, C L R_{3 m}=62 \%, C L R_{4 m}=67 \%$, 


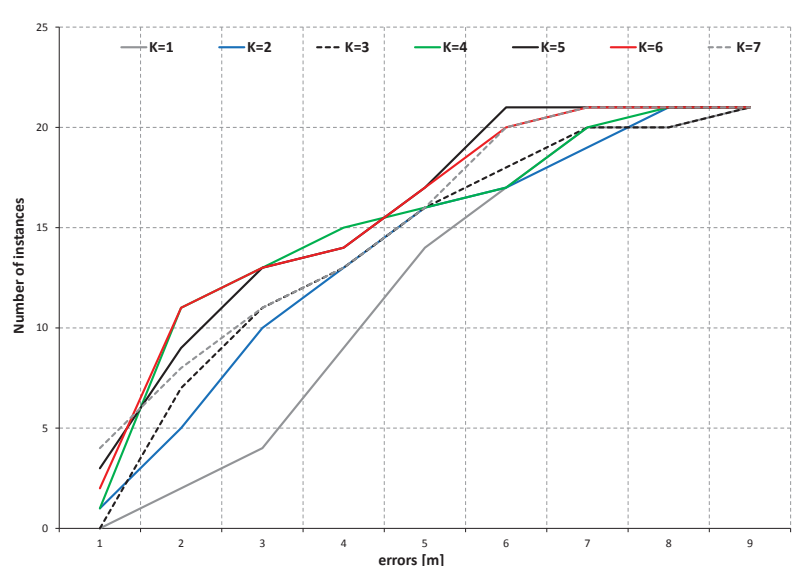

Figure 12: Isis' Temple: Errors.

$C L R_{5 m}=81 \%$, and $C L R_{6 m}=100 \%$. On average, the algorithm has a similar behavior in both the environments. Structural and architectural characteristics of the museum caused a higher attenuation of signals in different rooms and therefore a better distinction of fingerprints. This kind of analysis also revealed that in historic that in historic structures such as MANN, it is often necessary to install one AP per room, because for each AP the signal can propagate itself for no more than two rooms. Different considerations arise if the museum is composed by very large rooms such as large exhibition areas.

\section{CONCLUSIONS}

In this paper we have investigated fingerprint and trilateration algorithms in two real-case studies. We aimed at comparing the two approaches in an office environment and at suggesting a specific approach for the museum environment. Experimental results have proven that trilateration shows better performances in terms of adaption to changes (e.g., an addition or a new AP). On the other hand, fingerprint shows better results in terms of precision and correctness. The application of our results to the National Archeological Museum of Naples confirmed the highest correct localization rate at room level (i.e., 96\%) and a mean error of visitor's position of $2.88 \mathrm{~m}$. The results also confirmed the possibility to implement a location-based service for museum visitors. Indeed, in future research, we are going to examine a visitor's experience in a large museum in order to define his/her real behavior and analyze the common patterns. Thus, by detecting the position of the users, the system will provide localization-based services in terms of customized contents related to the rooms and artworks they admired during their visit to the museum.

\section{ACKNOWLEDGMENTS}

This work was partially supported by the Italian Ministry of Education, University and Research (MIUR) under the FIBAC Project PON01-02705.

\section{REFERENCES}

[1] A. Antoniou and G. Lepouras. Modeling visitors' profiles: A study to investigate adaptation aspects for museum learning technologies. J. Comput. Cult. Herit., 3(2):7:1-7:19, Oct. 2010.

[2] A. Au, C. Feng, S. Valaee, S. Reyes, S. Sorour, S. Markowitz, D. Gold, K. Gordon, and M. Eizenman. Indoor tracking and navigation using received signal strength and compressive sensing on a mobile device. Mobile Computing, IEEE Transactions on, 12(10):2050-2062, Oct 2013.

[3] K. H. Goldman. Cell phones and exhibitions 2.0: Moving beyond the pilot stage. In $J$. Trant and $D$. Bearman (eds.). Museums and the Web 2007: Proceedings, Toronto: Archives and Museum Informatics, March 2007.

[4] J. Krumm. Ubiquitous Computing Fundamentals. Chapman \& Hall/CRC, 1st edition, 2009.

[5] T. Kuflik, J. Kay, and B. Kummerfeld. Lifelong personalized museum experiences. In Pervasive User Modeling and Personalization (PUMP'10) at UMAP2010, pages 9-16, 2010.

[6] B. Li, J. Salter, A. G. Dempster, and C. Rizos. Indoor positioning techniques based on wireless lan. In Proc. of 1st Int. Conf. on Wireless Broadband and Ultra Wideband Communications. Auswireless 2006, pages 13-16, March 2006.

[7] H. Liu, H. Darabi, P. Banerjee, and J. Liu. Survey of wireless indoor positioning techniques and systems. Systems, Man, and Cybernetics, Part C: Applications and Reviews, IEEE Transactions on, 37(6):1067-1080, Nov 2007.

[8] A. Marota. Il museo come evento culturale: riflessioni e prospettive nell'era del digitale. In I. Pezzini and P. Cervelli, editors, Scene del Consumo: Dallo Shopping al Museo, pages 307-342. Collana Meltemi, 2007.

[9] C. Rohrig and F. KuİLnemund. Estimation of position and orientation of mobile systems in a wireless lan. In Decision and Control, 2007 46th IEEE Conference on, pages 4932-4937, Dec 2007.

[10] A. Roxin, J. Gaber, M. Wack, and A. Nait-Sidi-Moh. Survey of wireless geolocation techniques. In Globecom Workshops, 2007 IEEE, pages 1-9, Nov 2007.

[11] A. Schmitz-Peiffer, A. Nuckelt, M. Middendorf, and M. Burazanis. A new navigation system for indoor positioning (inLite). In Indoor Positioning and Indoor Navigation (IPIN), 2010 International Conference on, pages $1-7$, Sept 2010.

[12] U. Shala and A. Rodriguez. Indoor positioning using sensor-fusion in android devices, 2011.

[13] L. Solima. Il museo in ascolto. Nuove strategie di comunicazione per $i$ musei statali. Rubbettino, 2012.

[14] J. Torres-Solis, T. H. Falk, and T. Chau. A review of indoor localization technologies: towards navigational assistance for topographical disorientation. Ambient Intelligence, Felix Jesus Villanueva Molina (Ed.), pages 51-85, 2010.

[15] D. Wessel and E. Mayr. Potentials and challenges of mobile media in museums. iJIM, 1(1), 2007. 